\title{
Successful Conservative Management of Neonatal Pneumopericardium
}

\section{ISSN: 2576-9200}

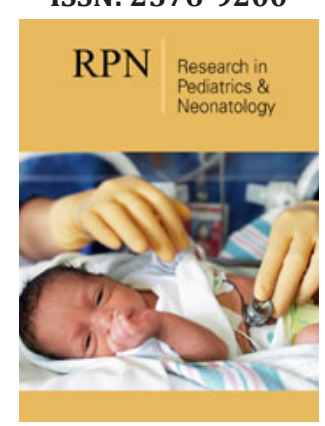

*Corresponding author: Chakrabarti R, Cloudnine Hospital, Haryana, India

Submission: 眥 February 05, 2020

Published: 泚 March 16, 2020

Volume 4 - Issue 3

How to cite this article: Chakrabarti R, Balde M, Kumar S, Yadav BS, Wazir S. Successful Conservative Management of Neonatal Pneumopericardium. Res Pediatr Neonatol. 4(3).RPN.000589.2020.

DOI: $10.31031 /$ RPN.2020.04.000589

Copyright $(\mathcal{C}$ Chakrabarti R. This article is distributed under the terms of the Creative Commons Attribution 4.0 International License, which permits unrestricted use and redistribution provided that the original author and source are credited.

\author{
Chakrabarti R*, Balde M, Kumar S, Yadav BS and Wazir S
}

Department of Neonatology, Cloudnine Hospital, Gurgaon, India

\begin{abstract}
Development of pneumo-pericardium in neonate is a rare event. If it occurs, it presents as a complication of respiratory distress syndrome. Commonly it requires some intervention to be cured, but we experienced a case which was totally resolved by conservative management. The baby was a late preterm and developed pneumopericardium at the age of 4 hours, he was managed conservatively. After 48 hours the pneumopericardium completely resolved.
\end{abstract}

Keywords: Neonate; Pneumopericardium; Mechanical ventilation; Conservative management

\section{Introduction}

Development of pneumo-pericardium in neonate is a rare event. If it occurs, it presents as a complication of respiratory distress syndrome. Commonly it requires some intervention to be cured, but we experienced a case which was totally resolved by conservative management (Figure 1).

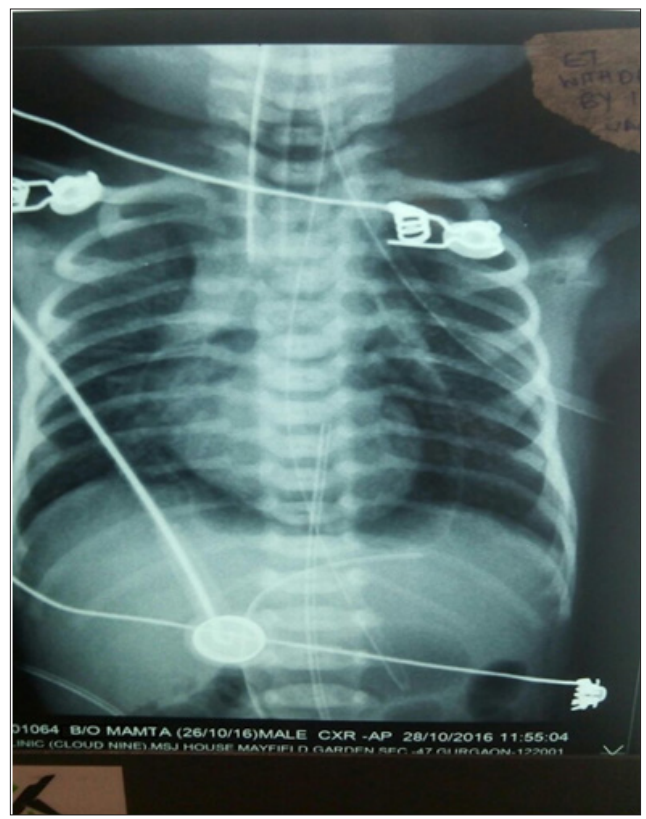

Figure 1: pneumopericardium.

\section{Case Report}

A 4 hours old 34 week late-preterm male baby presented with severe respiratory distress. Though he was vigorous at birth had meconium stained liquor and soon after birth, developed breathing difficulty. Because of severe distress and high fractional inspirational Oxygen $\left(\mathrm{FiO}_{2}\right)$ requirement he was put under mechanical ventilation and soon he required high frequency oscillatory ventilation (HFOV). His echocardiogram confirmed persistent pulmonary hypertension. As his sepsis screen was positive and blood culture confirmed Klebsiella, (but cerebrospinal fluid culture was sterile), he was kept under antibiotics and required inotrop support. 
Although initially his state improved with HFOV, on the second day there was a sudden increase of $\mathrm{FiO}_{2}$ requirement along with decrease in total leucocyte and platelet count. His chest X ray (CXR) showed pneumo-pericardium, but there was no feature of tamponade or tension pneumothorax. The baby was managed conservatively with antibiotics, mechanical ventilation and inotrops. CXR was realized 48 hours after the onset of pneumopericardium, it showed a complete resolution of the pneumopericardium, simultaneously his ventilator dependence decreased, after 72 hours of the incidence, the baby could be weaned off from HFOV to nasal intermittent mandatory ventilation. This was continued for 3 days. Before final shift to room air he was weaned to high flow nasal cannula, which was required for $24 \mathrm{hrs}$. He could be discharged on day 11 after admission, remained stable on follow up and was thriving well on follow up (Figure 2).

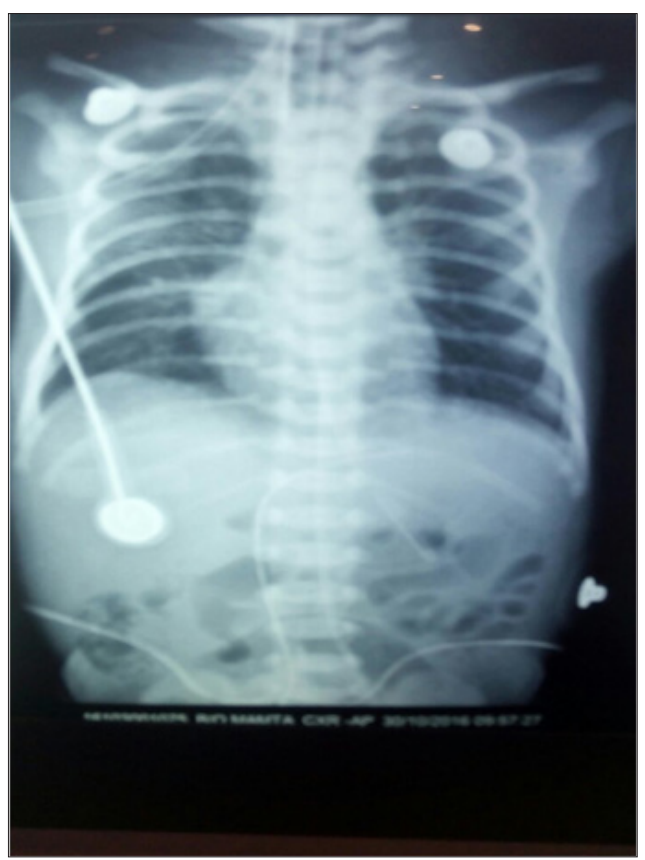

Figure 2: Post resolution.
Neonatal pneumopericardium is a rare, generally fatal condition [1] occurs mostly as a complication of ventilator management of respiratory distress syndrome [2] or meconium aspiration syndrome [3]. Isolated pneumopericardium without pneumomediastinum is even more uncommon [4]. Early recognition and prompt surgical intervention (preventing further accumulation of air by doing decompression through drainage) is the main stay of management to prevent fatal outcome. Conservative management, which we followed, becomes successful in very rare cases and possible in only isolated cases [4].

\section{References}

1. Karadžić K, Antović A, Ilić G, Banović LK (2007) Pneumopericardium: A possible rare cause of neonatal death. Medicine and Biology 14(2): 98100.

2. Emery RW, Foker J, Thompson TR (1984) Neonatal pneumopericardium: A surgical emergency. Ann Thorac Surg 37(2): 128-132.

3. Fellous L, Tourneux P, Pepin RB, Goissen C, Krim G (2005) Pneumopericardium: A rare complication of meconium aspiration syndrome. Arch Pediatr 12(1): 83.

4. Van Nostrand C, Beamish WE, Schiff D (1975) Neonatal pneumopericardium. Can Med Assoc J 112(2): 186-187. 